\title{
Dynamical Gluon Mass in QCD Processes
}

\author{
M. B. Gay Ducati and W. Sauter \\ (1) Grupo de Fenomenologia de Partículas de Altas Energias (GFPAE), \\ Instituto de Física, Universidade Federal do Rio Grande do Sul, \\ Porto Alegre, RS, Brazil
}

\section{Received on 25 September, 2006}

\begin{abstract}
We perform phenomenological applications of modified gluon propagators and running coupling constants in scattering processes in Quantum Chromodynamics (QCD). The modified forms of propagators and running coupling constant are obtained by non-perturbative methods. The processes investigated includes the diffractive ones - proton-proton elastic scattering, light vector meson photo-production and double vector meson production in gamma-gamma scattering - as well as the pion and kaon meson form factors. The results are compared with experimental data (if available), showing a good agreement with a gluon with dynamical mass but do not indicate the correct gluon propagator functional form.
\end{abstract}

Keywords: Pomeron; Elastic scattering; Non-perturbative gluons

\section{INTRODUCTION}

The challenging description of the infrared limit of Quantum Chromodynamics (QCD) is a field of investigation with important open questions. In the infrared, we need nonperturbative tools of investigation, as Schwinger-Dyson equations and lattice field theory, to obtain the Green functions to avoid the poles found in this kinematic region. For example, the gluon propagator (as well as any Green function) can have a different behavior in the infrared. With direct relation with the Green function, the running coupling constant can have a frozen value when the momentum goes to zero.

The diffractive processes have important contributions from the infrared. This class of high energy processes is described by the Pomeron exchange. A picture for the Pomeron which includes the non-perturbative effects is the LandshoffNachtmann (LN) model [1], where these effects are encoded in the Green functions, modified in the infrared kinematic region. The LN is employed in the description of proton-proton elastic scattering and vector meson photo-production. Another processes can be described by the use of modified gluon propagators, as for example, the meson form factors.

To obtain the gluon propagator to describe the above processes, some approximations are required. A consequence is that different solutions found in the literature following different approximation. Our goal in this work is to compare the distinct solutions of the gluon propagator with the experimental data on different processes. These different solutions includes: Cornwall's solution [2],

$$
D_{C}^{-1}\left(\mathbf{q}^{2}\right)=\left[\mathbf{q}^{2}+m_{C}^{2}\left(\mathbf{q}^{2}\right)\right] b g^{2} \ln \left(\frac{\mathbf{q}^{2}+4 m_{C}^{2}\left(\mathbf{q}^{2}\right)}{\Lambda_{\mathrm{QCD}}^{2}}\right),
$$

with

$$
m_{C}^{2}\left(\mathbf{q}^{2}\right)=m_{g}^{2}\left[\ln \left(\frac{\mathbf{q}^{2}+4 m_{g}^{2}}{\Lambda_{\mathrm{QCD}}^{2}}\right) / \ln \left(\frac{4 m_{g}^{2}}{\Lambda_{\mathrm{QCD}}^{2}}\right)\right]^{-12 / 11} ;
$$

Häbel et al. solution [3, 4]:

$$
D_{H}\left(\mathbf{q}^{2}\right)=\frac{\mathbf{q}^{2}}{\mathbf{q}^{4}+b^{4}}
$$

Gorbar and Natale solution [5]:

$$
\left[D_{G N}\left(\mathbf{q}^{2}\right)\right]^{-1}=\mathbf{q}^{2}+\mu_{g}^{2} \Theta\left(\xi^{\prime} \mu_{g}^{2}-\mathbf{q}^{2}\right)+\frac{\mu_{g}^{4}}{\mathbf{q}^{2}} \Theta\left(\mathbf{q}^{2}-\xi^{\prime} \mu_{g}^{2}\right),
$$

where $\Theta$ is the step function, $\mu_{g}=0.61149 \mathrm{GeV}$ and $\xi^{\prime}=$ 0.9666797; Aguilar and Natale solution [6]:

$$
D_{A N}\left(\mathbf{q}^{2}\right)=\frac{1}{\mathbf{q}^{2}+\mathcal{M}^{2}\left(\mathbf{q}^{2}\right)}, \quad \mathcal{M}^{2}\left(\mathbf{q}^{2}\right)=\frac{m_{0}^{4}}{\mathbf{q}^{2}+m_{0}^{2}},
$$

where $m_{0}^{2}=0.99 \mathrm{GeV}^{2}$ for the QCD scale $\Lambda_{\mathrm{QCD}}=335 \mathrm{MeV}$; Alkofer and collaborators solution [7-10]:

$$
D_{A L}\left(\mathbf{q}^{2}\right)=\frac{\omega}{\mathbf{q}^{2}}\left[\frac{\mathbf{q}^{2}}{\Lambda_{\mathrm{QCD}}^{2}+\mathbf{q}^{2}}\right]^{2 \kappa}\left(\alpha_{S}^{(\mathrm{AL})}\left(\mathbf{q}^{2}\right)\right)^{-\gamma},
$$

where $\omega=2.5, \Lambda_{\mathrm{QCD}}=510 \mathrm{MeV}, \kappa \approx 0.595, \gamma=-13 / 22$ and $\alpha_{s}^{(\mathrm{AL})}\left(\mathbf{q}^{2}\right)$ is the running coupling constant (see below). The solutions are displayed in the Fig. 1 .

The running coupling have a straight connection with the modified Green functions. The expressions are related with the propagator employed. The analytical expressions are: Cornwall $[2,11]$,

$$
\alpha_{s}\left(k^{2}\right)=4 \pi / \beta_{0} \ln \left(\frac{k^{2}+\xi_{m_{p}}^{2}\left(k^{2}\right)}{\Lambda_{\mathrm{QCD}}^{2}}\right),
$$

where $\xi \approx 4$ and $m_{\mathcal{P}}\left(k^{2}\right)$ is the massive term of the gluon propagator; Alkofer expression

$$
\begin{aligned}
\alpha_{s}^{(\mathrm{AL})}\left(\mathbf{q}^{2}\right) & =\frac{1}{1+\left(\mathbf{q}^{2} / \Lambda_{\mathrm{QCD}}^{2}\right)}\left[\alpha_{s}(0)+\frac{4 \pi}{\beta_{0}} \frac{\mathbf{q}^{2}}{\Lambda_{\mathrm{QCD}}^{2}}\right. \\
& \left.\times\left(\frac{1}{\ln \left(\mathbf{q}^{2} / \Lambda_{\mathrm{QCD}}^{2}\right)}+\frac{1}{1-\left(\mathbf{q}^{2} / \Lambda_{\mathrm{QCD}}^{2}\right)}\right)\right],
\end{aligned}
$$

where $\alpha_{s}(0) \approx 2.972$ and $\beta_{0}=11$. The different solutions are displayed in the Fig. 2 


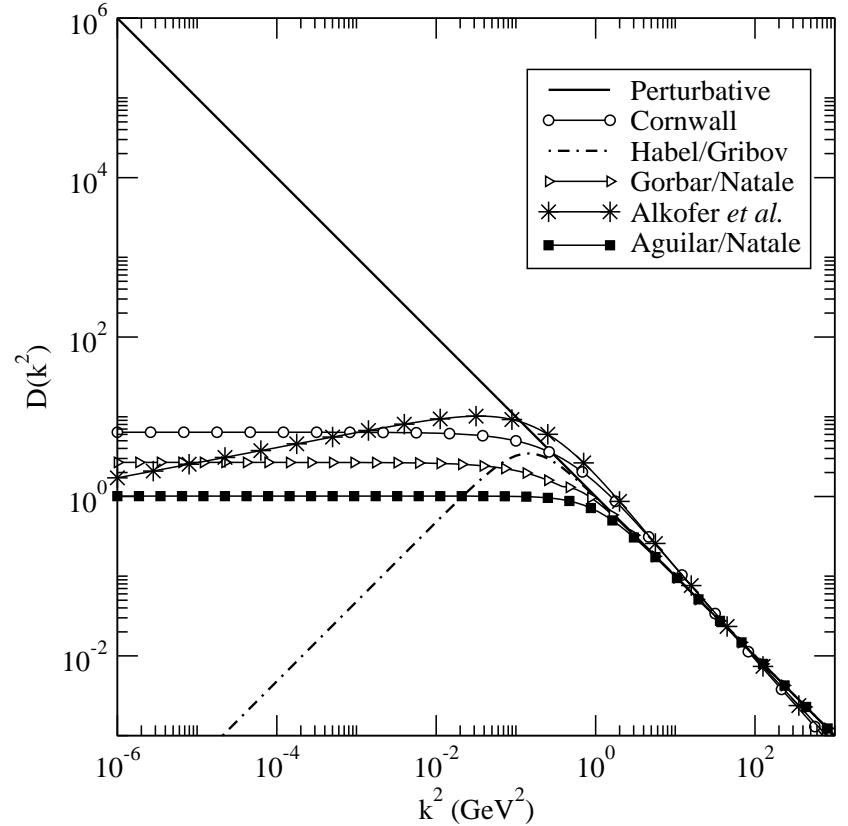

FIG. 1: Distinct modified gluon propagators compared with the perturbative one. The propagators are indicated in the plot legend.

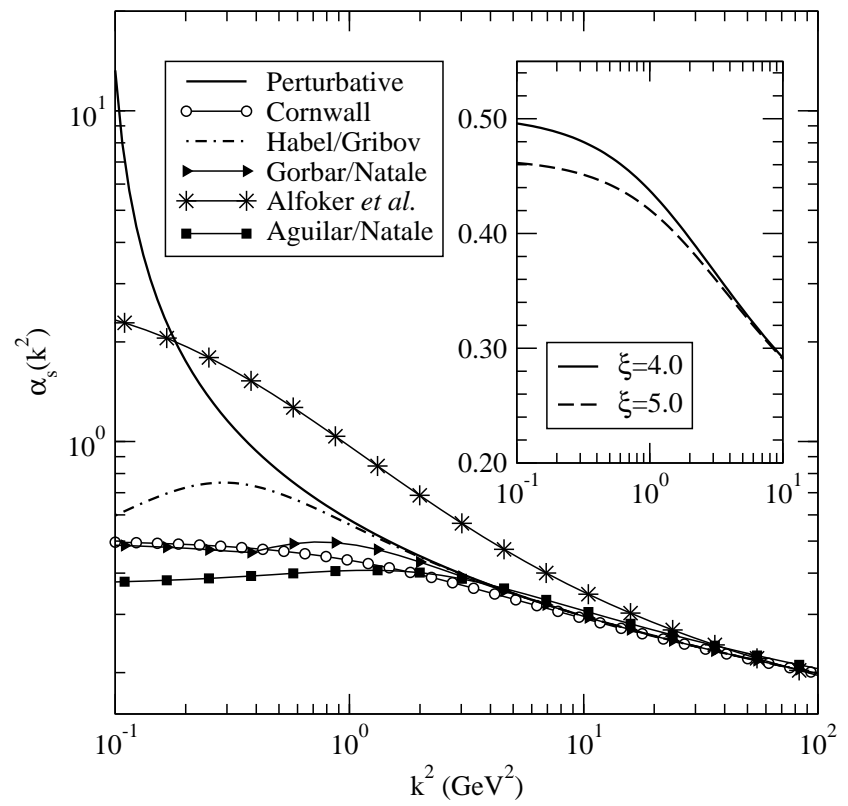

FIG. 2: Comparison between different results for the running coupling constant. In the detail, two results of the Cornwall frozen coupling with two different $\xi$ 's.

\section{ELASTIC $p p$ SCATTERING}

To compare the gluon propagator above with the experimental data for the elastic $p p$ scattering, we employ a modified scattering amplitude (based in [12-14]) to include frozen running coupling and Regge trajectory of the soft Pomeron, namely,

$$
\begin{aligned}
& \mathcal{A}_{2}^{p p}(s, t)=8 i s\left(\frac{s}{s_{0}}\right)^{\alpha_{\mathbb{P}}(t)-1} \int d^{2} \mathbf{k} \alpha_{s}\left(\frac{\mathbf{q}}{2}+\mathbf{k}\right) \mathcal{D}\left(\frac{\mathbf{q}}{2}+\mathbf{k}\right) \\
& \times \alpha_{s}\left(\frac{\mathbf{q}}{2}-\mathbf{k}\right) \mathcal{D}\left(\frac{\mathbf{q}}{2}-\mathbf{k}\right)\left[G_{p}(q, 0)-G_{p}\left(q, k-\frac{q}{2}\right)\right]^{2} .
\end{aligned}
$$

where $\alpha_{\mathbb{P}}(t)=\alpha(0)+\alpha^{\prime}(t)$ is the (soft) Pomeron trajectory (with $\alpha(0)=1.08$ and $\left.\alpha^{\prime}(t) \simeq 0.25 \mathrm{GeV}^{-2}[15]\right), G_{p}(q, k)$ is the convolution of proton cross-sections, related with the Dirac form factor of the proton,

$$
\begin{aligned}
G_{p}(q, 0) & =F_{1}\left(q^{2}\right) \\
G_{p}\left(q, k-\frac{q}{2}\right) & =F_{1}\left(q^{2}+9\left|k^{2}-\frac{q^{2}}{4}\right|\right) .
\end{aligned}
$$

The cross section are given by the optical theorem,

$$
\sigma_{\mathrm{tot}}=\frac{\mathcal{A}_{2}^{p p}(s, 0)}{i s}, \quad \frac{d \sigma}{d t}=\frac{\left|\mathcal{A}_{2}^{p p}(s, t)\right|^{2}}{16 \pi s^{2}}
$$

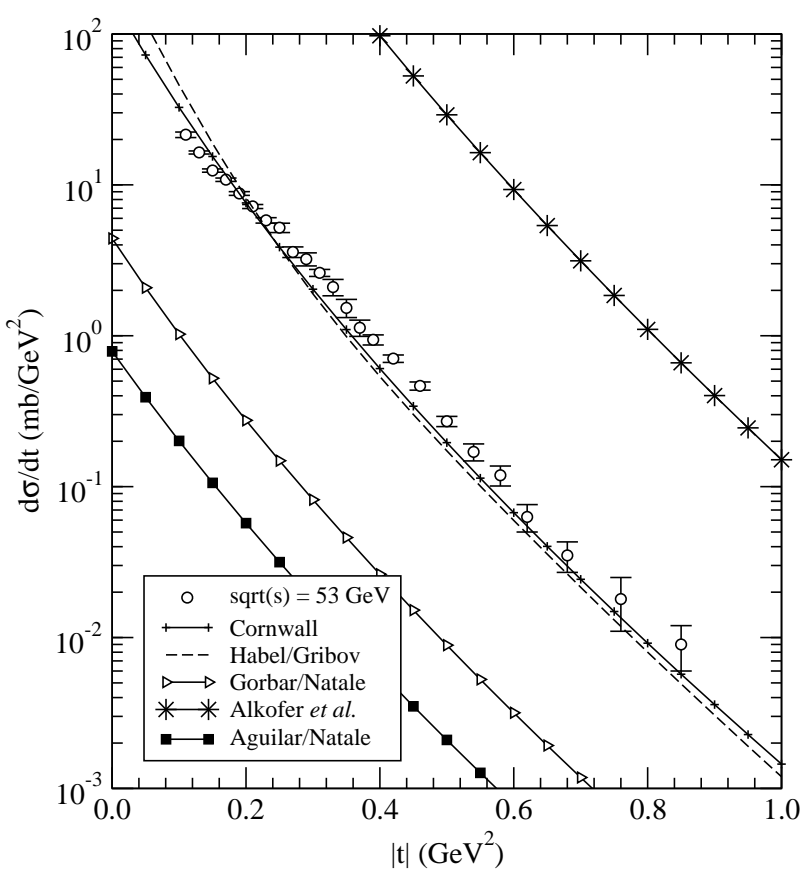

FIG. 3: Results of the modified model, fitting to the $p p$ elastic scattering data with $\sqrt{s}=53 \mathrm{GeV}$ [16] with different gluon propagators and frozen coupling constants.

\section{LIGHT MESON DIFFRACTIVE PHOTO-PRODUCTION}

The ideas employed above can be applied in the light vector meson production. This process is a good laboratory for the Pomeron exchange. The modified amplitudes are obtained in 
the same spirit of the amplitude in the above section, based in Cudell/Royen model [17]. The differential cross section reads

$$
\frac{d \sigma}{d t}=\frac{1}{16 \pi} C^{2} \zeta^{2\left(\alpha_{\mathbb{P}}(t)-1\right)} f^{2}\left(\Delta, Q^{2}\right)\left[1+\varepsilon \frac{Q^{2}}{m_{V}^{2}}\right],
$$

where $\zeta=s / s_{0}$ with $s_{0}=Q^{2}+m_{V}^{2}-t, \quad \mathcal{C}=$ $(64 / \sqrt{6}) g_{\mathrm{elm}}^{V} m_{V} \sqrt{m_{V} f_{V}}, \varepsilon \approx 1$ is the polarization of the photon beam,

$$
\begin{aligned}
f\left(\Delta, Q^{2}\right)= & \int d^{2} \mathbf{k} \alpha_{s}(\mathbf{k}) \alpha_{S}(\mathbf{k}-\Delta) \mathcal{D}(\mathbf{k}) \mathcal{D}(\mathbf{k}-\Delta) \\
& \times \frac{\left[F_{1}(t)-\mathcal{E}_{2}(k, k-\Delta)\right][\mathbf{k} \cdot(\mathbf{k}-\Delta)]}{t-m_{V}^{2}-Q^{2}+4 \mathbf{k} \cdot(\mathbf{k}-\Delta)}
\end{aligned}
$$

where $F_{1}(t)$ is the Dirac proton form factor and $\mathcal{E}_{2}(k, k-\Delta)=$ $F_{1}\left(\mathbf{k}^{2}+(\mathbf{k}-\Delta)^{2}-\mathbf{k} \cdot(\mathbf{k}-\Delta)\right)$. The massive parameters are the same as in the $p p$ elastic scattering.

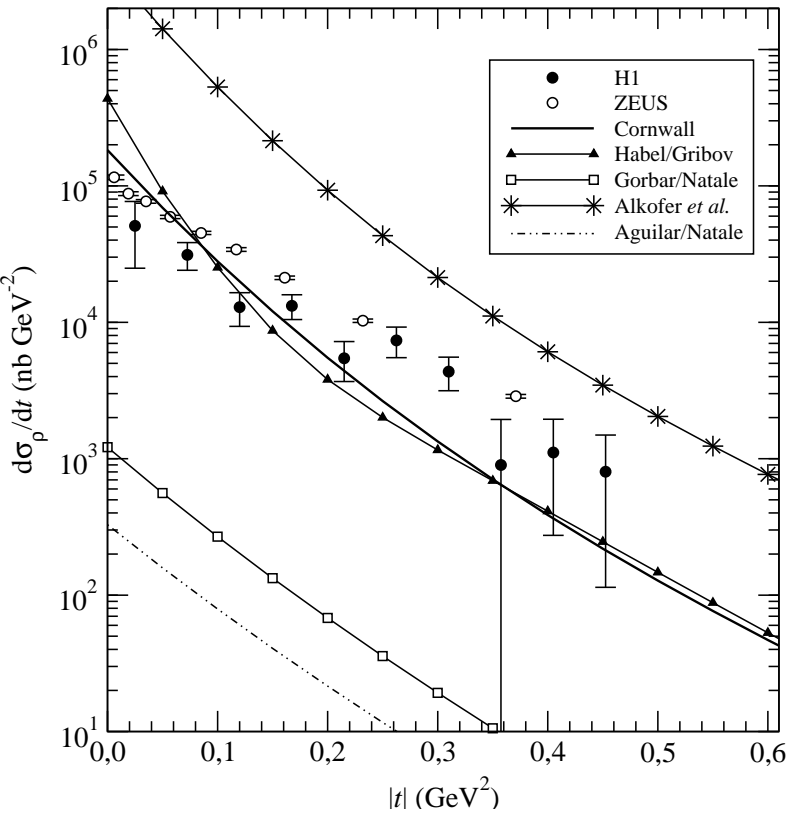

FIG. 4: $\rho$ photo-production differential cross section, compared with the $\mathrm{H} 1$ and ZEUS data $[18,19]$

\section{DOUBLE $J / \Psi$ PRODUCTION IN $\gamma \gamma$ SCATTERING}

The double diffractive production of meson vectors in photon-photon collisions is a clean test for the BFKL Pomeron, due the hard scale provided by the mass of the meson and/or the large momentum transfer. The experimental possibilities includes CLIC and LHC in the peripheral heavy ion collisions. The scattering amplitude in the Born level (two gluon exchange is [20]

$\left.\mathfrak{I} m \mathcal{A}^{(0)}(s, t)=\int \frac{d^{2} \mathbf{k}}{\pi}\left[\Phi_{0}\left(k^{2}, Q^{2}\right)\right]^{2} D(\mathbf{k}+\mathbf{Q} / 2) D(\mathbf{k}-\mathbf{Q} / 2) \emptyset 14\right)$ where the impact transition $\gamma J / \Psi$ factor is

$$
\Phi_{0}\left(k^{2}, Q^{2}\right)=\frac{C}{2} \sqrt{\alpha_{e m}} \alpha_{s}\left(\mu^{2}\right)\left[\frac{1}{\bar{q}^{2}}-\frac{1}{m_{J / \Psi}^{2} / 4+k^{2}}\right],
$$

with $\mathcal{C}=q_{c} \frac{8}{3} \pi m_{J / \Psi} f_{J / \Psi}, q_{c}=\frac{2}{3}, f_{J / \Psi}=0.38 \mathrm{GeV}, \bar{q}^{2}=$ $m_{J / \Psi}^{2}+\frac{Q^{2}}{4}, \mu^{2}=k^{2}+Q^{2} / 4+\left(m_{J / \Psi} / 2\right)^{2}$. Improvements and applications are made in $[21,22]$ with the inclusion of full BFKL amplitude and applications in other processes.

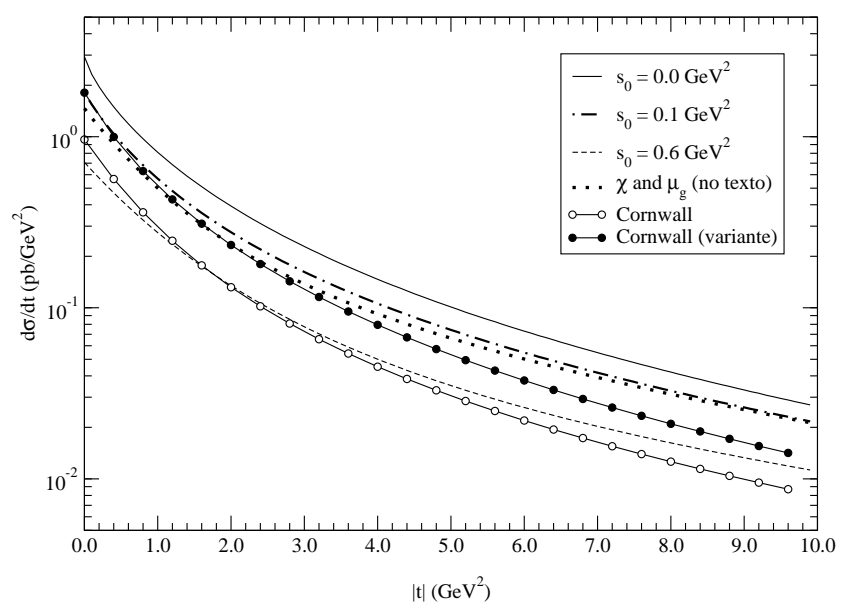

FIG. 5: Differential cross section for $\gamma \gamma \rightarrow J / \psi J / \psi$ using Gorbar/Natabe, Cornwall gluon propagators.

\section{MESON FORM FACTORS}

The meson form factors measure the hadron charge distribution, defined by

$$
\begin{gathered}
\frac{d \sigma}{d \Omega}(e+\pi, K \rightarrow e+\pi, K)=\left(\frac{d \sigma}{d \Omega}\right)_{\text {point }}\left|F_{\pi, K}\left(Q^{2}\right)\right|^{2} . \\
F_{M}\left(Q^{2}\right)=\int_{0}^{1} d x \int_{0}^{1} d y \phi_{M}^{*}\left(y, \tilde{Q}_{y}\right) T_{H}\left(x, y, Q^{2}\right) \phi_{M}\left(x, \tilde{Q}_{x}\right),
\end{gathered}
$$

where $T_{H}$ is the hard scattering amplitude in LO, modified to include non-perturbative contributions (see ,for example, [23]),

$$
T_{H}\left(x, y, Q^{2}\right)=\frac{64 \pi}{3}\left\{\frac{2}{3} \tilde{\alpha}_{s}\left(\hat{k}^{2}\right) D\left(\hat{k}^{2}\right)+\frac{1}{3} \tilde{\alpha}_{s}\left(\hat{p}^{2}\right) D\left(\hat{p}^{2}\right)\right\},
$$

$\phi_{M}$ is the distribution amplitude given by Bethe-Salpeter evolution equation (also modified to include a frozen running coupling),

$\phi_{M}\left(x, Q^{2}\right)=x(1-x) \sum_{n=0}^{\infty} C_{n}^{(3 / 2)}(2 x-1)\left[\frac{\alpha_{s}\left(Q^{2}\right)}{\alpha_{S}\left(Q_{0}^{2}\right)}\right]^{d_{n}} \phi_{n}^{(M)}\left(Q_{0}^{2}\right)$. 


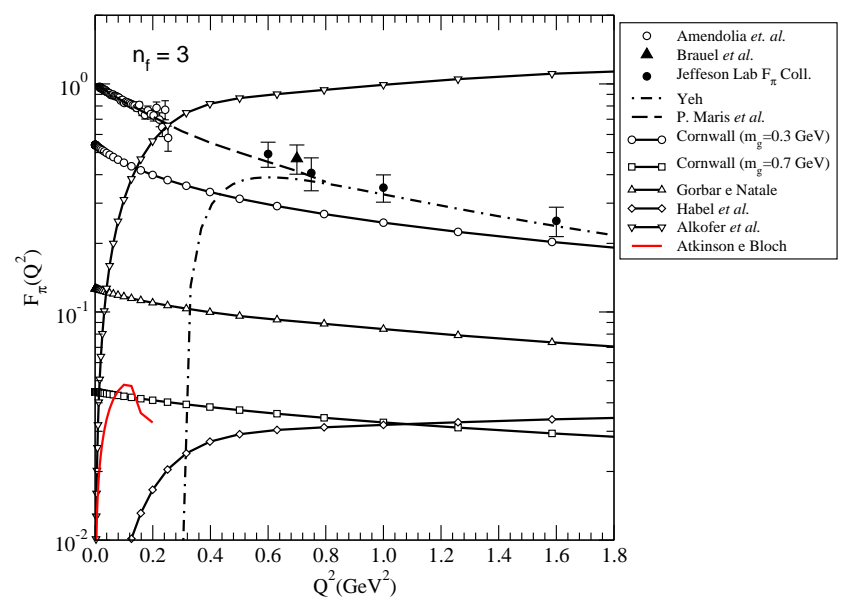

FIG. 6: Pion form factor with different propagators in comparison with the data (see [20]).

\section{CONCLUSIONS}

From the results displayed above, we conclude that the use of a modified gluon propagator in the description of processes in which soft kinematic scales are relevant is useful. We use a simple model (two gluon exchange) of diffractive scattering with modifications to include modified gluon propagators. The models allows modifications to include the alternative IR forms of gluon propagator, also in the case of meson form factors. The massive parameters of the propagator and coupling constant are kept fixed in the original values in the diffractive processes, giving different results for different processes. In the case of meson form factors, a fit to the experimental data is performed, giving different values for the parameters, but fitting the data. Despite the crudeness of the model employed in the description of the data, the comparison of the results from different models is a (possible) useful filter for different forms of the gluon propagators. Due to the (possible) dependence on the model employed in the process, our results favor solutions with a dynamical gluon mass, although do not discriminate which is the best choice among the propagators employed here.

\section{ACKNOWLEDGMENTS}

The authors thanks A. Natale for the discussions and appointments along this work.

(1993).

[1] P. V. Landshoff and O. Nachtmann. Z. Phys. C 35 , 405 (1987)

[2] J. M. Cornwall. Phys. Rev. D 26, 1453 (1982).

[3] U. Häbel, R. Könning, H.-G. Reusch, M. Stingl, and S. Wigard. Z. Phys. A 336, 423 (1990).

[4] U. Häbel, R. Könning, H.-G. Reusch, M. Stingl, and S. Wigard. Z. Phys. A 336, 435 (1990).

[5] E. V. Gorbar and A. A. Natale. Phys. Rev. D 61, 054012 (2000).

[6] A. C. Aguilar and A. A. Natale. JHEP 08, 057 (2004).

[7] R. Alkofer and L. Von Smekal. Phys. Rep. 353, 281 (2001).

[8] L. von Smekal, A. Hauck, and R. Alkofer. Phys. Rev. Lett. 79, 3591 (1997).

[9] C. S. Fisher and R. Alkofer. Phys. Rev. D 67, 094020 (2003).

[10] R. Alkofer, W. Detmold, C. S. Fisher, and P. Maris. Nucl. Phys. Proc. Suppl. 141, 122 (2005).

[11] J. Papavassiliou and J. M. Cornwall. Phys. Rev. D 44, 1285 (1991). x

[12] J. R. Cudell and D. A. Ross. Nucl. Phys. B 359, 247 (1991).

[13] F. Halzen, G. Krein, and A. A. Natale. Phys. Rev. D. 47, 295

[14] D. S. Henty, C. Parrinello, and D. G. Richards. Phys. Lett. B 369, 130 (1996).

[15] A. Donnachie and P. V. Landshoff. Phys. Lett. B 296, 227 (1992).

[16] A. Breakstone et al. Nucl. Phys. B 248, 253 (1984)

[17] J. R. Cudell and I. Royen. Phys. Lett. B 397, 317 (1997).

[18] J. Adloff et al. Eur. Phys. J. C 13, 371 (2000).

[19] J. Breitweg et al. Eur. Phys. J. C 6, 603 (1999).

[20] M. B. Gay Ducati and W. K. Sauter. Phys. Lett. B 521, 259 (2001).

[21] V. P. Gonçalves and W. K. Sauter. Eur. Phys. J. C 44, 515 (2005).

[22] V. P. Goncalves, M. V. T. Machado, and W. K. Sauter. Eur. Phys. J. C 46, 219 (2006)

[23] M. B. Gay Ducati and W. K. Sauter. Phys. Rev. D 67, 014014 (2003). 